\title{
The Influence Factor Research of Fiber-Optic Sensors Based on Surface
}

\section{Plasmon}

\author{
Fu Li-hui ${ }^{1, \text { a }}$, Dai Junfeng ${ }^{2, b}$ \\ ${ }^{1}$ Faculty of Automation, Huaiyin Institute of Technology,Jiangsu Huaian,223003,China \\ ${ }^{2}$ Faculty of Electronic Information Engineering,Huaiyin Institute of Technology,Jiangsu \\ Huaian,223003,China

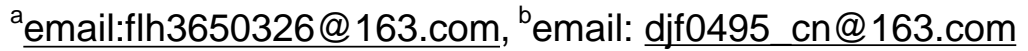

\begin{abstract}
Key Words:Optical fiber; sensor; surface plasmon resonance (SPR); refractive index; resonance wavelength; MATLAB
\end{abstract}

Abstract:Based on the theory of Surface Plasmon Resonance(SPR), the technique of Surface Plasmon Resonance was described and a detailed mechanism of the Fiber-Optic sensing has been discussed.The influence of the real and imaginary part of metal membrane'dielectric constant,the thickness of the metal,the refraction index of the fiber core and environment medium,on SPR reflective spectral properties were simulated by MATLAB.The main performance parameters of SPR sensors are respectively discussed.Finally,based on the research of influential factor in inspiring SPR phenomenon and the change regularities of reflective spectrum with performance parameters, the feasibility of wavelength modulating SPR Sensors is discussed.It provide a theoretic guide for improving the sensitivity and accuracy of Surface Plasmon Resonance(SPR) sensor,and the optimization design of fiber SPR sensor system.

\section{Introduction}

The resonance phenomenon of the plasma resonance sensor is based on the interface between light and the electrolyte, which is caused by the resonance of the free electrons under some conditions[1]. The evanescent wave generated by the total internal reflection of the light at the interface of the glass causes the surface plasma.The plasma [2,3] is a kind of non charged ionized material of ions and electrons and the core particle, namely the quantum of surface charge oscillations.Metal is a low temperature plasma [4],its charge density is high, the valence electron of metal' oscillation relative to positive ion is similar to the plasma oscillation of conductive gas ,so it is called metal surface plasma wave.The evanescent waves realize the oscillation. When the beam goes through from the optically denser medium into the thinner medium,it is divided into a transmitted light and a reflected light, at this time, if the incident angle is larger than the critical angle, there will be total reflection phenomenon, in this process, there are still electromagnetic wave in optically thinner medium, the amplitude of the electromagnetic wave will increase with the penetration depth in exponential decay, which is the evanescent wave [5]. When the incident angle or wavelength is a proper value, leading to the frequency and wave number of metal surface plasma wave is equal to the evanescent wave,at this time, some of the energy of the incident light is absorbed and the reflected light energy declined sharply, the lowest reflecting strength of resonance peaks in reflectance spectra appear, and when the medium refraction rate close to the surface of a metal film is different, it'resonance peak position will change, which is the basic principle of SPR sensor used to detect the medium parameters based on surface plasmon resonance technology. 
SPR technology has a very large potential value and broad application prospect in food safety determination, environmental waste water and waste gas monitoring, therefore, the researchers all over the world pay attention to SPR[1-5]. However, the actual application of SPR sensor is not optimistic, foreign commercial SPR sensors is expensive, while domestic research still stays in the experimental stage, the main reasion is the influence factors of surface plasmon resonance phenomenon is numerous,so the sensitivity of the sensor needs to be further improved . In view of this phenomenon, this paper discusse the influencing factors of plasma resonance, through the study the influence law of the imaginary and real part of complex dielectric constant of metal film, the thickness of the metal,the refractive index of optical fiber core, the refractive index of the medium on surface plasmon resonance spectroscopy, which provides theoretical guidance for the further optimization of the better sensor SPR sensor design.

\section{The theoretical calculation of optical fiber SPR sensor}

The basic structure of optical fiber surface plasmon resonance sensor consists of three layers: the core, the sensitive film and the environment medium. In practice, the optical fiber SPR sensing device often adopt the sensing device proposed by Jorgenson. One is the online transmission, the other is the terminal reflection. The terminal reflection measurement structure consists of sensing head by deposition metal film on optical fiber core, total reflection phenomenon occurs in the interface of fiber core and cladding by excitation light, and cause surface plasmon resonance,through detecting the distribution relationship between the reflected light intensity and wavelength in the end of fiber,the quantitative analysis of measured medium can be realized. This single total reflection calculation is similar to that of the prism type sensor. According to the properties of the plasma wave, the magnetic field distribution can be:

$$
H_{y}(x)=\left\{\begin{array}{l}
A_{2} e^{k_{p z}\left(x-d_{m}\right)}+B_{2} e^{-k_{p z}\left(x-d_{m}\right)} \mathrm{K}\left(x>d_{m}\right) \\
A_{1} e^{k_{m z} x}+B_{1} e^{-k_{m z} x} \mathrm{~K}\left(0<x<d_{m}\right) \\
A_{0} e^{k_{s z} x} \mathrm{~K}(x<0)
\end{array}\right.
$$

Thereinto, $\quad k_{j z}=\left(k^{2} \varepsilon_{j}-k_{x}^{2}\right)^{1 / 2}(j=p, m, s), \quad k_{x}=k \sqrt{\varepsilon_{p}} \sin \theta$

Using the continuous conditions $H_{y}$ and $\frac{1}{\varepsilon} H_{y}$ of the TM wave at the two boundaries, there is

When $x=0,\left\{\begin{array}{l}A_{0}=A_{1}+B_{1} \\ \frac{\varepsilon_{m} k_{s z}}{\varepsilon_{s} k_{m z}} A_{0}=A_{1}-B_{1}\end{array}\right.$

$$
\text { When } x=d_{m},\left\{\begin{array}{l}
A_{1} e^{k_{m z} d_{m}}+B_{1} e^{-k_{m z} d_{m}}=A_{2}+B_{2} \\
\frac{\varepsilon_{P} k_{m z}}{\varepsilon_{m} k_{p z}}\left[A_{1} e^{k_{m z} d_{m}}-B_{1} e^{-k_{m z} d_{m}}\right]=A_{2}-B_{2}
\end{array}\right.
$$

Thus, the reflectivity formula[10] is: 


$$
R=\left|\frac{B_{2}}{A_{2}}\right|^{2}=\left|\frac{r_{p m}+r_{m s} e^{+2 i k_{m z} d_{m}}}{1+r_{p m} r_{m s} e^{+2 i k_{m z} d_{m}}}\right|^{2}
$$

Thereinto, $r_{p m}=\frac{k_{p z} \varepsilon_{m}-k_{m z} \varepsilon_{p}}{k_{p z} \varepsilon_{m}+k_{m z} \varepsilon_{p}}, \quad r_{m s}=\frac{k_{m z} \varepsilon_{s}-k_{s z} \varepsilon_{m}}{k_{m z} \varepsilon_{s}+k_{s z} \varepsilon_{m}}, \quad k=\frac{\omega}{c}$

Thereinto, ${ }^{\varepsilon_{s}}$ is the dielectric constant of sample, $\varepsilon_{m}$ is the dielectric constant of metal clad, $\varepsilon_{p}$ is the dielectric constant of core; $\mathrm{k}$ is the wave number in vacuum, ${ }_{m}$ is the thickness of metal layer, $\omega$ the angular frequency of excitation light, $c$ is the velocity of light in a vacuum.

\section{The simulation analysis of SPR'sensing characteristics}

Establish the simulation experiment of optical fiber SPR sensor'single reflection, according to the reflectance model shown in the formula(4), after determining the relevant experimental parameters, the relationship between reflectivity and other specific parameters can be calculated, so as to study the influence of various factors on the performance of optical fiber SPR sensor.

\section{The effect of complex permittivity of metal film on SPR spectra}

In the process of MATLAB simulation, the influence of the imaginary part and the real part of metal film 'dielectric constant on the resonance wavelength is investigated.

The influence of the real part of metal film'complex dielectric constant on SPR reflection spectrum

The main parameters of the experiment are as follows: the thickness of the film $d=60$, the wavelength lang=300:5:700, the dielectric constant of the core yibu0 $=0.9$, the dielectric constant of the measured medium yibu2=1.5; the incident angle shita=60; the imaginary part of metal'dielectric constant is 0.07 .The wavelength modulation SPR'reflection spectrum of gold film'complex permittivity is calculated, as shown in Fig 1.

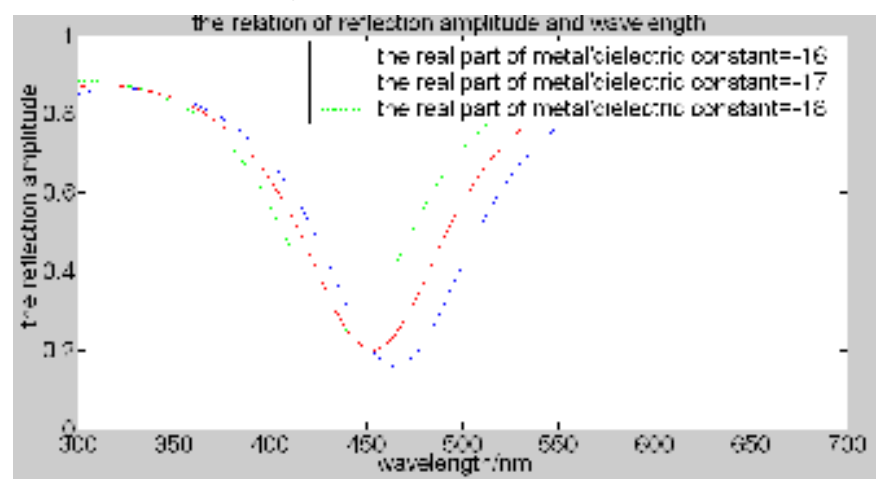

Fig.1The theoretical effect curve of the real part of metal membrane'dielectric constant on SPR spectral

Fig 1 shows that the gold film'real part of complex permittivity gradually reduce from $-16,-17$ to -18 , the resonant wavelength and minimum reflectance has a certain change, but in comparison, the biggest influence is on the resonant wavelength, it gradually decreased by $470 \mathrm{~nm}, 460 \mathrm{~nm}, 440 \mathrm{~nm}$.

The influence of the imaginary part of metal film'complex dielectric constant on SPR reflection spectrum

The real part of metal film'dielectric constant is -18 , the other parameters are the same as experiment 1 . The wavelength modulation SPR reflection spectrum is calculated, as shown in Fig 2. 


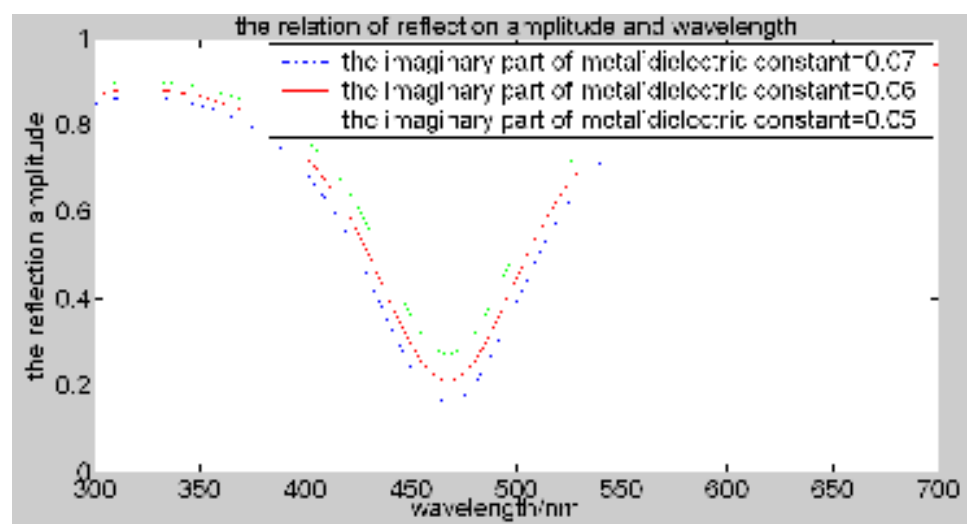

Fig.2 The theoretical effect curve of the imaginary part of metal membrane'dielectric constant on SPR spectral

Fig 2 shows that the imaginary part of gold film'complex permittivity reduce by $0.07,0.06,0.05$, the resonance wavelength has little change with around $480 \mathrm{~nm}$, but the reflex amplitude changed greatly, it decrease with the imaginary part of the dielectric constant of gold film. thus, the detection sensitivity reduced gradually.

From the above, the metal film'dielectric constant has great influence on the resonant wavelength, because the actual metal'complex dielectric constant change with the input light' wavelength, the chemical stability, reliability and other factors of different metal film will affect the application of SPR, therefore, in the actual measurement process, in addition to consider the measurement'sensitivity, it also need to consider the above factors to choose suitable metal film.

\section{The effect of metal film'thickness on SPR spectra}

The dielectric constant of the metal film is $-18+0.07 i$, the other parameters are the same as experiment 1. Calculate the wavelength modulation SPR reflection spectrum with of different thickness of the metal layer, as shown in fig 3.

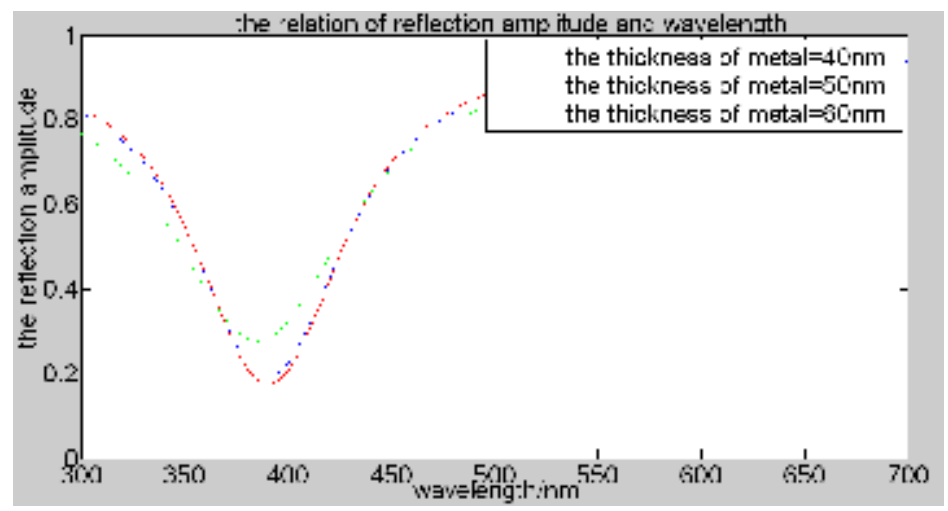

Fig.3 The theoretical effect curve of the thickness of metal on SPR reflective spectral

Fig 3 shows that the thickness of the metal layer increase by 40nm, 50nm, $60 \mathrm{~nm}$, the reflex amplitude change greatly,basically decreases with the increasing of the thickness of metal layer,but it exist the optimum film thickness.In this experiment, when the film thickness is $50 \mathrm{~nm}$, the light energy'attenuation is the strongest,so,the sensitivity is highest.The main reason is that when the thickness is small, such as 40nm, part of the energy will penetrate through the metal layer,so the SPR effect is not obvious, when the film thickness is large, such as 60nm, it is not easy for evanescent wave to inspire the SPR effect. 


\section{The effect of fiber core'refractive index on SPR spectra}

The dielectric constant of metal is $-18+0.07$, the thickness is $50 \mathrm{~nm}$, and the other parameters are the same as experiment 1. The reflection spectrum of wavelength modulation SPR is calculated, as shown in fig 4.

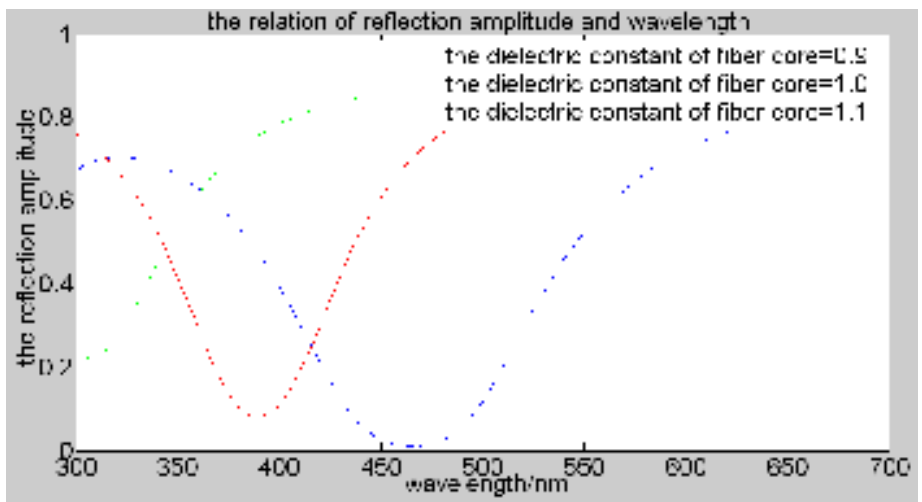

Fig.4 The theoretical effect curve of the dielectric constant of fiber core on SPR reflective spectral

Fig 4 shows that the dielectric constant of fiber core increase by $0.9,1,1.1$, the resonance wavelength increase gradually from $310 \mathrm{~nm}$ to $480 \mathrm{~nm}$, and when the dielectric constant of fiber core is 0.9 , the reflection amplitude is the minimum,so it is easy to detect.

\section{The effect of refractive index of environmental medium on SPR spectra}

The dielectric constant of the fiber core is 0.9 , and the other parameters are the same as experiment 3. Calculate the reflection spectrum of wavelength modulation SPR, as shown in fig 5

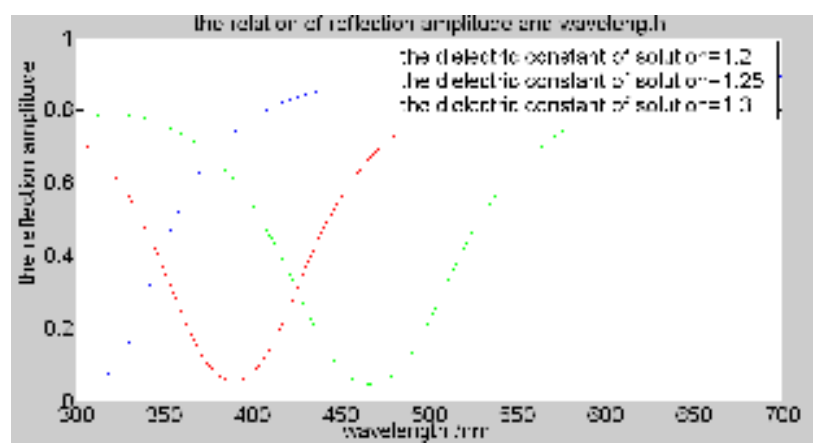

Fig.5 The theoretical effect curve of the dielectric constant of environment medium on SPR spectral the reflection amplitude

Fig 5 shows that the dielectric constant of measured solution increase by 1.2, 1.25, 1.3, the resonance wavelength increase gradually by $320 \mathrm{~nm}, 400 \mathrm{~nm}, 480 \mathrm{~nm}$, but the reflection spectrum is basically unchanged, so,there is a good linear relationship between the dielectric constant of solution and the resonant wavelength, and the dielectric constant varies with the nature and concentration of the solution, therefore, the measurement information of solution. can be obtained by the change of resonance wavelength.

\section{Conclusion}

Through the establishment the model of reflectance spectral,analyse the affect characteristics of the optical fiber SPR'main parameters of sensor in theory,get the change rule of reflection intensity with the dielectric constant of metal film,the thickness of the metal, the dielectric constant of fiber core,dielectric constant of the environment.The study found that the attenuation of the optical fiber SPR sensor'resonance wavelength is an important basis for determining the measurement 
sensitivity, and the above parameters have different effects on it, therefore, in actual measurement, it need to select the appropriate type of metal film,thickness and fiber type, in order to improve the performance of the SPR sensor.

\section{References}

[1]Nylander C,Liedberg B,Lind T.Gas detection by means of surface plasmon resonance[J].Sensor and actuators, 1982,(3):79-88.

[2]Stefan Lofaas,Malmqvist M,Ronnberg I.Bioanalysis with surface plasmon resonance[J].Sensor and actuators, 1991,(5):79-84.

[3]Wang J,Wang L.Y.,Sun Y.,Zhu X.N,Cao Y B.,Wang X.H.,Zhang H.Q,Song D.Q.Surface plasmon resonance biosensor based on $\mathrm{Au}$ nanoparticle in titania solgel membrane[J].Colloid Surf.B,2010,78:520-525.

[4]S.Wang,M.Chiu,J.Hsu,R.Chang,F.Wang.Theoretical analysis and experimental evaluation of D-type optical fiber sensor with a thin gold film[J].Optics Communications,2005,253:283-289.

[5]Tang,J.L.,Cheng,S.F.,Hsu,W.T.,Chiang,T.Y.,Chau,L.K.Fiber-optic biochemical sensing with a colloid gold-modified long period fiber grating[J].Sens.and Actua.B,2006,119:105-109. 